\title{
Treatment of Eyelid Ptosis due to Kearns-Sayre Syndrome Using Frontalis Suspension
}

\author{
Laurenz Weitgasser, Gottfried Wechselberger, Florian Ensat, Rene Kaplan, Michaela Hladik \\ Department of Plastic, Aesthetic, and Reconstructive Surgery, Krankenhaus Barmherzige Brueder Salzburg, Teaching Hospital of the \\ Paracelsus Medical University, Salzburg, Austria
}

\begin{abstract}
Blepharoptosis is a common indication for surgery in plastic surgery units, yet its possible underlying pathology frequently remains unidentified. A 52-year-old man with a 20-year history of progressive bilateral ptosis (right > left) presented with recurrent ptosis of both eyes; he had undergone an operation on the levator aponeurosis 12 years prior. Due to the suspicion of an underlying disease, he was evaluated further. Chronic progressive external ophthalmoplegia in transition to the more severe syndromic variant Kearns-Sayre syndrome, a mitochondrial disorder causing myopathy, was diagnosed. The patient was treated with coenzyme $\mathbf{0 1 0}$, and he underwent ptosis surgery on both eyes. This case illustrates a potentially multi-systemic disease that was diagnosed by a further evaluation of a common symptom, in this case worsening blepharoptosis. Awareness of myopathic symptoms is necessary to prevent overlooking serious yet improvable conditions.
\end{abstract}

Keywords Blepharoptosis / Kearns-Sayre syndrome / Chronic progressive external opthalmoplegia / Blepharoplasty

\author{
Correspondence: Laurenz Weitgasser \\ Department of Plastic, Aesthetic, and \\ Reconstructive Surgery, Klinik für \\ Hand-, Mikro- und Rekonstruktive \\ Brustchirurgie, Marienhospital \\ Stuttgart, teaching Hospital of the \\ University of Tuebingen, \\ Boeheimstraße 37, Stuttgart 70199, \\ Germany \\ Tel: +49711-64890-7216 \\ Fax: +49711-64890-8222 \\ E-mail: Laurenz.weitgasser@pmu. \\ ac.at
}

No potential conflict of interest relevant to this article was reported.

Received: 24 Sep 2014 • Revised: 15 Dec 2014 • Accepted: 23 Dec 2014

pISSN: 2234-6163 • elSSN: 2234-6171 • http://dx.doi.org/10.5999/aps.2015.42.2.214 • Arch Plast Surg 2015;42:214-217

\section{INTRODUCTION}

Kearns-Sayre syndrome (KSS), first described in 1958 [1], is rare and results from a mitochondrial disorder. Few cases of similar blepharoplastic procedures in patients suffering from chronic progressive external ophthalmoplegia (CPEO) or KSS have been reported to date $[2,3]$. This case illustrates a very common condition in plastic surgery underlying an uncommon disease that is unfamiliar to plastic surgeons. Moreover, controversy remains regarding the optimal surgical procedure. Here, we present our case and review and discuss the relevant literature.

\section{CASE}

A 52-year-old man with a 20-year history of progressive bilateral blepharoptosis (Fig. 1A) who underwent a levator aponeurosis operation on the right side 12 years prior presented with recurrent ptosis of both eyes, with a greater defect on the right side. The eyelid excursion was $2 \mathrm{~mm}$ on the right side and $4 \mathrm{~mm}$ on the left side preoperatively (Fig. 1B). Other than his ptosis, the man, who was of small stature, underwent the first operation when his friends noticed that he suddenly looked tired and looked like he had grown older. However, one year after the surgery, he already had a remarkable recurrence of his ptosis, whereupon a detailed investigation was undertaken to identify the underlying disease. Funduscopic findings resembled retinitis pigmentosa. Electromyography showed no abnormal spontaneous activity, but a few action potentials with reduced amplitude were detected. Light microscopy of a biopsy from the deltoid muscle showed single ragged red fibers, and enzyme histochemistry demonstrat- 


\section{Fig. 1. Case presentation}

$(A, B)$ A 52-year-old male patient with a 20-year history of progressive bilateral blepharoptosis who had recurrent blepharoptosis after a previous levator aponeurosis operation on the right side 12 years earlier.
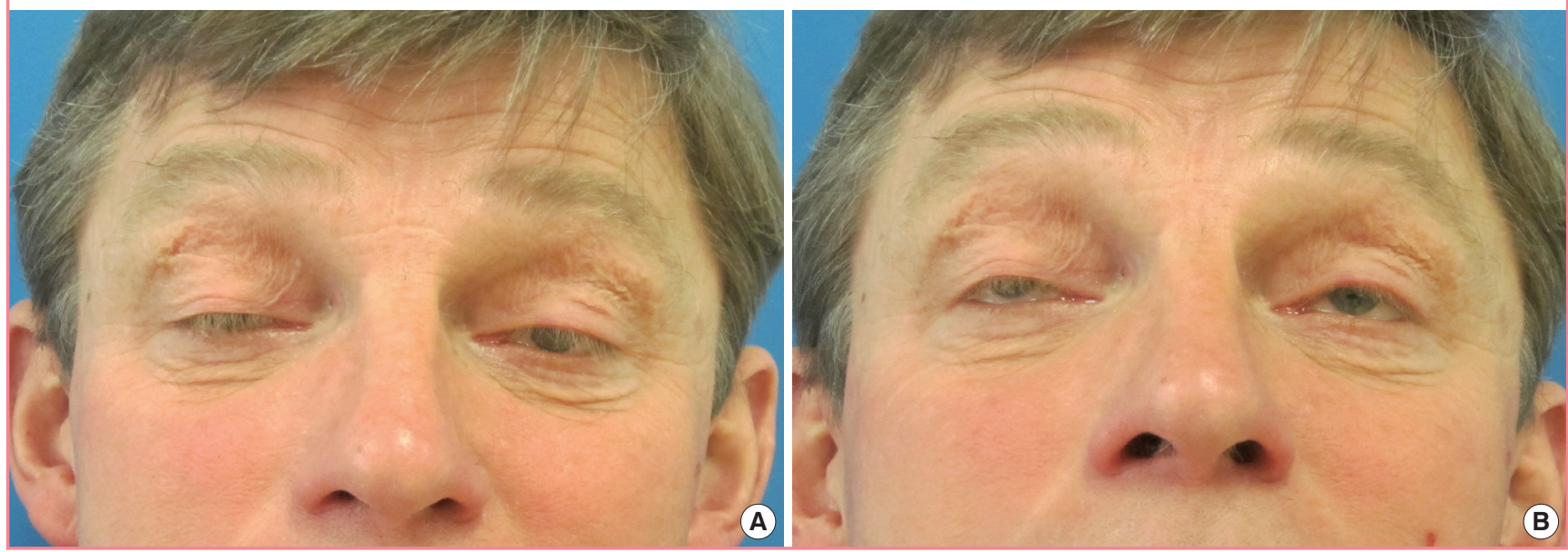

\section{Fig. 2. Intraoperative view}

$(A, B)$ Tunnels under the eyebrow were prepared with thin cannulas. Flexor carpi radialis tendon strips were guided through the tunnels as autologous sling material for frontalis suspension.

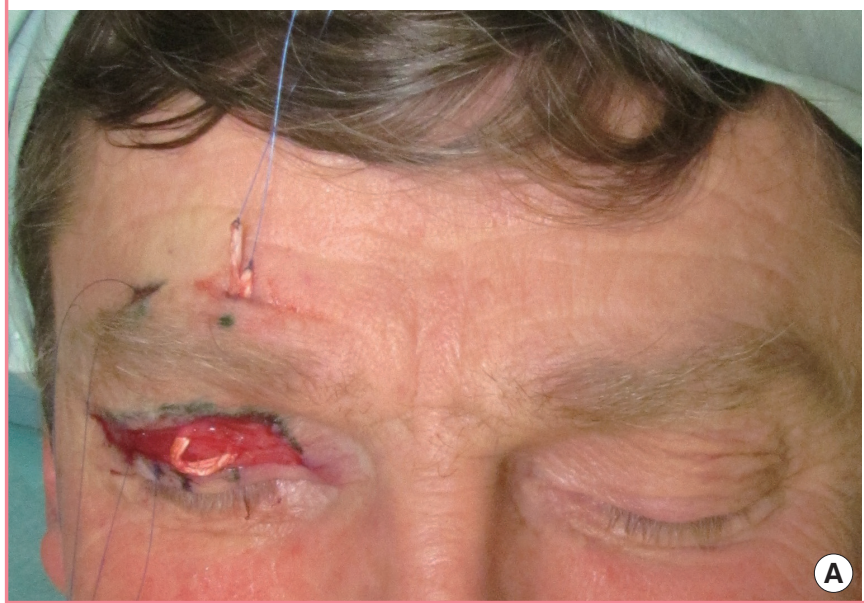

ed cytochrome oxidase-negative fibers. Hematological investigations were normal apart from mildly increased lactate $\left(2.5 \times 10^{9} /\right.$ $\mathrm{L}$; upper limit of normal, $\left.2.0 \times 10^{9} / \mathrm{L}\right)$ and total protein in the cerebrospinal fluid (CSF, $45.2 \mathrm{mg} / \mathrm{dL}$; upper limit of normal, $43.0 \mathrm{mg} / \mathrm{dL})$.

The diagnosis of KSS was made. Over the next 5 years, the patient's ptosis worsened to the extent that it prevented him from safely operating a vehicle or performing his job as a cabinet maker. Additionally, he relied on hearing aids, as his hearing had also declined in recent years. Therefore, blepharoplasty and frontalis suspension were performed on both eyes. The first operation was performed on the right side, and the left side was reconstructed two weeks later. Additionally, the patient received continuous coenzyme Q10 treatment to prevent further disease progression. Regular follow-up and symptomatic and supportive

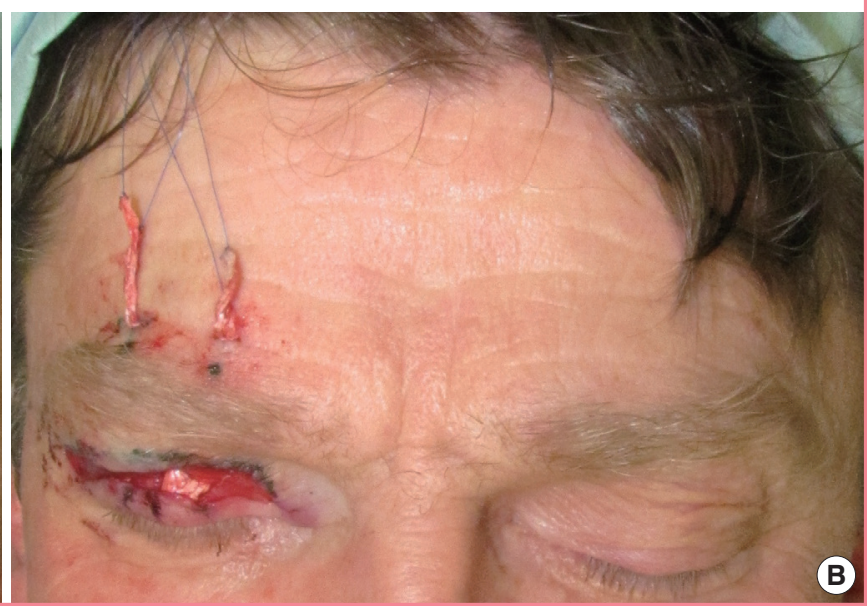

care were provided.

Subsequent ptosis surgery of the right eye involved the harvesting of the flexor carpi radialis (FCR) tendon (the palmaris longus tendon was missing) from the left forearm and using it as an autologous sling material for frontalis suspension surgery. Therefore, a 100-mm-long and 3-mm-wide piece of the FCR tendon was harvested from the right forearm and subsequently separated into two 100-mm-long and 1.5-mm-wide strips. Excess skin was excised and removed, and upper eyelid blepharoplasty was performed. Additionally, three $10-\mathrm{mm}$-long incisions were made symmetrically above the right eyebrow, and three tunnels under the eyebrow were prepared with thin cannulas. The four ends of the prepared strips were then guided through the three tunnels (Fig. 2A). While the lower part of the sling was anchored at the tarsus using 6-0 prolene, the four ends were join- 
Fig. 3. Immediate postoperative result

(A, B) Immediate postoperative result, after skin closure and successful ptosis adjustment of the right eye with frontalis suspension.
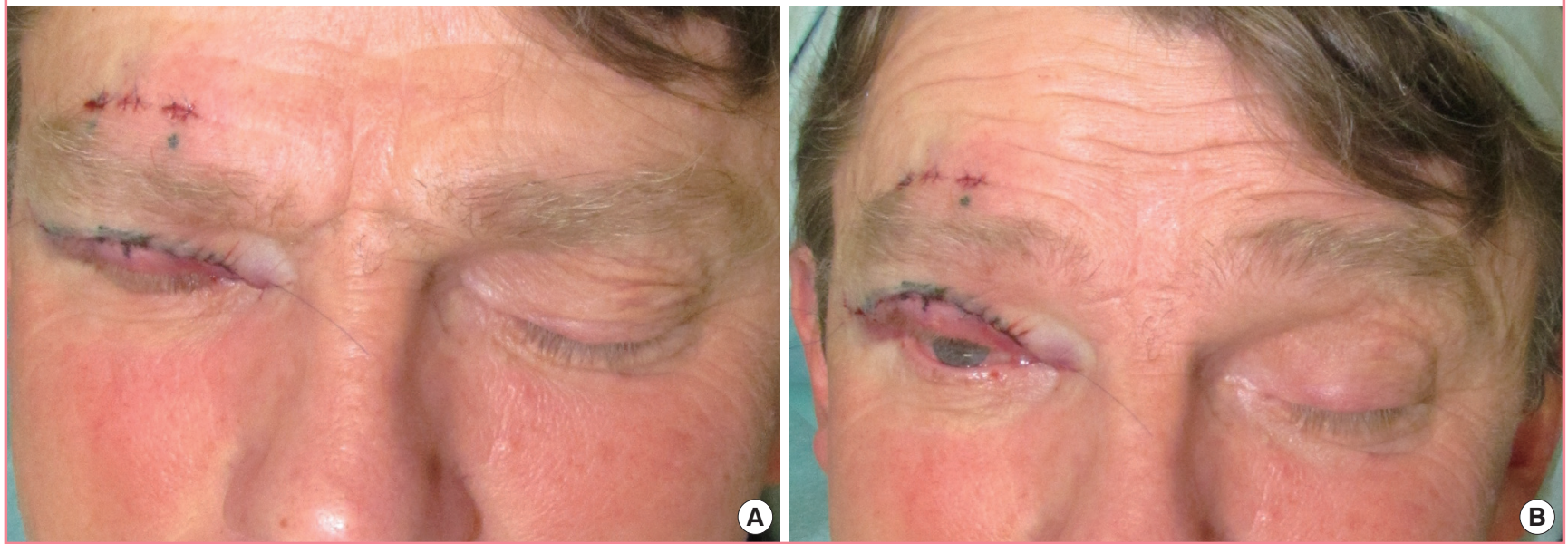

Fig. 4. Postoperative results

(A) Results 6 months postoperatively. (B) Results 12 months postoperatively.
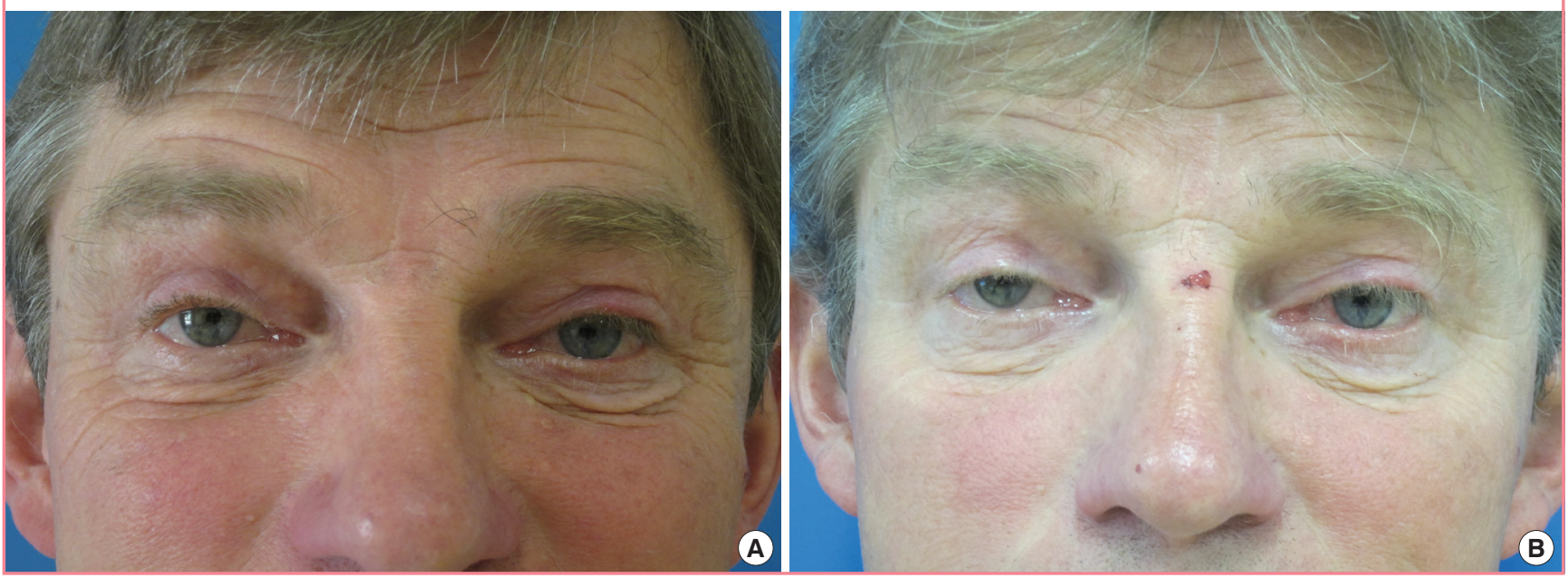

ed with 5-0 prolene superiorly, creating two circular slings and thereby adjusting the ptosis (Fig. 2B). The skin was closed with subcuticular continuous 6-0 prolene sutures, and the wounds were dressed.

Immediate postoperative eyelid excursion was $12 \mathrm{~mm}$ on the right eye and $14 \mathrm{~mm}$ after the second surgery on the left eye (Fig. 3). Six-month postoperative follow-up showed eyelid excursion of $11 \mathrm{~mm}$ on the right eye and $14 \mathrm{~mm}$ on the left eye (Fig. 4A). One-year postoperative follow-up still showed excellent results with eyelid excursion of $11 \mathrm{~mm}$ on the right eye and $14 \mathrm{~mm}$ on the left eye without lagophthalmos. The patient was very pleased and was able to take a job as a taxi driver and continue working (Fig. 4B). To continue evaluating the course of the disease, the patient is currently under the ongoing care of an internist.

\section{DISCUSSION}

CPEO is not rare as compared to KSS. Nonetheless, most plastic surgeons are unfamiliar with these disorders. KSS was first described in 1958 as the simultaneous appearance of external ophthalmoplegia with pigmentary degeneration of the retina, heart block, and skeletal myopathy underlying a mitochondrial disorder $[1,4]$. It sporadically develops in early adolescence and is a rarely observed syndrome, but the true incidence is unknown. Bilateral ptosis is often an early symptom. Additional findings can include short stature, cerebellar dysfunction, increased CSF protein levels, sensorineural hearing loss, and diabetes. Because these symptoms can be associated with a wide range of diseases, many investigations and a multidisciplinary team of medical specialists are required to thoroughly evaluate these patients. In- 
vestigations include cranial magnetic resonance imaging, the tension test, ophthalmoscopy, electromyography, and nerve conduction velocity measurement as well as the analysis of blood, urine, and CSF. If substantial suspicion for KSS exists, muscle biopsy, electrocardiography, a treadmill stress test, and detailed endocrine testing must be performed. A molecular genetic workup of mitochondrial DNA mutations allows an accurate diagnosis. The syndrome is slowly progressive with a variable prognosis depending on the severity of the symptoms. At present, treatment is very limited and narrowed to symptomatic and supportive care [3]. Limited progression and improvement of symptoms can be achieved by the systemic administration of coenzyme Q10 [5].

Here, we described recurrent bilateral blepharoptosis after previous surgical treatment in a 52-year-old patient. The reoccurrence of blepharoptosis could have been caused by either disease progression or insufficient previous surgery. To date, few surgical treatments for blepharoptosis in KSS have been reported $[2,3,6]$. Some ophthalmology studies have been reported, but in these studies, the surgical procedure for correcting eyelid ptosis was always secondary, or no long-term data were available. In 2007, Papageorgiou et al. [3] presented a case of a 16-yearold patient with blepharoptosis due to KSS who underwent a levator advancement procedure. Although they reported a good result 18 months postoperatively, the functional loss of the levator palpebrae muscle cannot be corrected using this method. The underlying problem is the myopathy that involves the levator and orbicularis oculi muscles, leading to ptosis and poor lid closure [6]. Levator advancement cannot adequately correct this problem; a shorter muscle-aponeurosis complex can be created, but the functional problem will remain. We believe that functional reconstruction, similar to frontalis suspension [7], will lead to the best result. Although the frontalis muscle is not entirely spared [8] by the disease, it appears to be less, and much later, affected and therefore, is useful for the frontalis suspension surgery. The levator palpebrae on the other hand is usually affected primarily and loses its entire function at a faster rate over time.

\section{REFERENCES}

1. Kearns TP, Sayre GP. Retinitis pigmentosa, external ophthalmophegia, and complete heart block: unusual syndrome with histologic study in one of two cases. AMA Arch Ophthalmol 1958;60:280-9.

2. Park SB, Ma KT, Kook KH, et al. Kearns-Sayre syndrome: 3 case reports and review of clinical feature. Yonsei Med J 2004; 45:727-35.

3. Papageorgiou G, Vlachos S, Tentis D. Blepharoptosis due to Kearns-Sayre syndrome. J Plast Reconstr Aesthet Surg 2008; 61:573-4.

4. Kearns TP. External ophthalmoplegia, pigmentary degeneration of the retina, and cardiomyopathy: a newly recognized syndrome. Trans Am Ophthalmol Soc 1965;63:559-625.

5. Ogasahara S, Nishikawa Y, Yorifuji S, et al. Treatment of Kearns-Sayre syndrome with coenzyme Q10. Neurology 1986; 36:45-53.

6. Eshaghian J, Anderson RL, Weingeist TA, et al. Orbicularis oculi muscle in chronic progressive external ophthalmoplegia. Arch Ophthalmol 1980;98:1070-3.

7. Salvi SM, Currie ZI. Frontalis suspension sling using palmaris longus tendon in chronic progressive external ophthalmoplegia. Ophthal Plast Reconstr Surg 2009;25:140-1.

8. Lane CM, Collin JR. Treatment of ptosis in chronic progressive external ophthalmoplegia. Br J Ophthalmol 1987;71: 290-4. 